\title{
Endemic Burkitt lymphoma: a complication of asymptomatic malaria in sub-Saharan Africa based on published literature and primary data from Uganda, Tanzania, and Kenya
}

Lawrence S. Redmond ${ }^{1}$, Martin D. Ogwang ${ }^{2,3}$, Patrick Kerchan ${ }^{3,4}$, Steven J. Reynolds ${ }^{5}$, Constance N. Tenge ${ }^{6}$, Pamela A. Were ${ }^{7}$, Robert T. Kuremu ${ }^{6}$, Nestory Masalu ${ }^{8}$, Esther Kawira ${ }^{9}$, Isaac Otim²,3, Ismail D. Legason 3,4, Herry Dhudha ${ }^{9}$, Leona W. Ayers ${ }^{10}$, Kishor Bhatia', James J. Goedert ${ }^{1}$ and Sam M. Mbulaiteye ${ }^{1 *}$ (C)

\begin{abstract}
Background: Endemic Burkitt lymphoma (eBL) is an aggressive B cell non-Hodgkin lymphoma associated with antigenic stimulation from Plasmodium falciparum malaria. Whether eBL risk is related to malaria parasite density is unknown. To address this issue, children with eBL, asymptomatic and clinical malaria, as a surrogate of malaria parasite density, were assessed.

Methods: Malaria-related laboratory results (parasite density, haemoglobin, platelet count, and white cell count [WBC]) count) were compiled for 4019 eBL cases and 80,532 subjects evaluated for asymptomatic malaria or clinical malaria (severe malaria anaemia, hyperparasitaemia, cerebral malaria, malaria prostration, moderate malaria, and mild malaria) in 21 representative studies published in Africa (mostly East Africa) and 850 eBL cases and 2878 controls with primary data from the Epidemiology of Burkitt Lymphoma in East African Children and Minors (EMBLEM) case-control study in Uganda, Tanzania, and Kenya. The average values of malaria-related laboratory results were computed by condition and trends across single-year age groups were assessed using regression and spline models.

Results: Overall, malaria infection or malaria was diagnosed in 37,089 of children compiled from the literature. Children with eBL and asymptomatic parasitaemia/antigenaemia, but not those with clinical malaria, were closest in their mean age (age 7.1-7.2 vs. 7.4-9.8 years), haemoglobin level (10.0-10.4 vs. 11.7-12.3 g/dL), malaria parasite density (2800 vs. $1827-7780$ parasites/ $\mu \mathrm{L}$ ), platelet count (347,000-353,000 vs. $244,000-306,000$ platelets/ $\mu \mathrm{L}$ ), and WBC count (8180-8890 vs. 7100-7410 cells/ML). Parasite density in these two groups peaked between four to five years, then decreased steadily thereafter; conversely, haemoglobin showed a corresponding increase with age. Children with clinical malaria were markedly different: all had an average age below 5 years, had dramatically elevated parasite density $(13,905-869,000$ parasites $/ \mu \mathrm{L})$ and dramatically decreased platelet count $(<159,000$ platelets $/ \mu \mathrm{L})$ and haemoglobin $(<7 \mathrm{~g} / \mathrm{dL})$.
\end{abstract}

*Correspondence: mbulaits@mail.nih.gov

${ }^{1}$ Division of Cancer Epidemiology and Genetics, National Cancer Institute, National Institutes of Health, Bethesda, MD, USA

Full list of author information is available at the end of the article

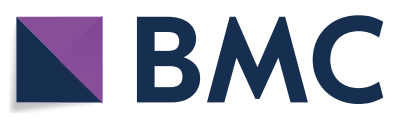

(c) The Author(s) 2020. This article is licensed under a Creative Commons Attribution 4.0 International License, which permits use, sharing, adaptation, distribution and reproduction in any medium or format, as long as you give appropriate credit to the original author(s) and the source, provide a link to the Creative Commons licence, and indicate if changes were made. The images or other third party material in this article are included in the article's Creative Commons licence, unless indicated otherwise in a credit line to the material. If material is not included in the article's Creative Commons licence and your intended use is not permitted by statutory regulation or exceeds the permitted use, you will need to obtain permission directly from the copyright holder. To view a copy of this licence, visit http://creativeco mmons.org/licenses/by/4.0/. The Creative Commons Public Domain Dedication waiver (http://creativecommons.org/publicdomain/ zero/1.0/) applies to the data made available in this article, unless otherwise stated in a credit line to the data. 
Conclusions: eBL and asymptomatic parasitaemia/antigenaemia, but not clinical malaria, were the most similar conditions with respect to mean age and malaria-related laboratory results. These results suggest that children with asymptomatic parasitaemia/antigenaemia may be the population at risk of eBL.

Keywords: Burkitt lymphoma (BL), Malaria complications, Plasmodium falciparum, Epstein-barr virus (EBV), East Africa

\section{Background}

Endemic Burkitt Lymphoma (eBL) is an aggressive B-cell non-Hodgkin lymphoma that is associated with endemic Plasmodium falciparum malaria [1]. Thus, the incidence of eBL correlates with the endemicity of $P$. falciparum malaria [1-3] and eBL incidence is highest in malaria endemic countries in sub-Saharan Africa [4], where eBL cases account for $50-75 \%$ of childhood cancers in some countries [5]. The role of malaria is supported by significant associations of eBL risk with high antibody titers of markers of long-term exposure to P. falciparum infection [6-8] and inverse associations with antibodies that are associated with protection from severe P. falciparum infection $[6,9]$. In addition, there is support from indirect evidence based on inverse associations with on carriage of genetic variants that are associated with resistance to severe malaria morbidity [10-12], especially the sickle cell trait [13]. However, these results, although important because they are not affected by reverse causality, have not been consistent because they were non-significant in some studies $[14,15]$ and null in at least one study [16]. The conflicting results may be due to small and under-powered studies or reliance on hospitalbased studies or selection bias of controls.

Whether the relationship between malaria and eBL is related to malaria morbidity and circulating malaria parasite burden and inflammation [17], for which morbidity is a surrogate of, is unknown. The severity of clinical malaria (severe malaria anaemia, hyperparasitaemia, cerebral malaria, malaria prostration, moderate malaria, and mild malaria) is directly related to parasite burden and associated host response [18], but the correlation between clinical malaria, which is a surrogate for uncontrolled parasite burden [17], has not been investigated.

This paper reports an investigation to assess the patterns of age and selected malaria-related laboratory measures (parasite density, haemoglobin, platelet count, and white cell count (WBC) count) in children with eBL, asymptomatic parasitaemia/antigenaemia, and clinical malaria in Uganda, Tanzania, and Kenya using primary data from a case-control study or secondary data extracted from papers published in malaria endemic areas.

\section{Methods}

Sources of subjects evaluated for malaria-related laboratory measures

The analysis utilized primary data compiled from children enrolled in the Epidemiology of Burkitt Lymphoma in East African Children and Minors (EMBLEM) Study conducted in Uganda, Tanzania, and Kenya during 2010-2016 [19]. The EMBLEM study enrolled children aged 0-15 years old with eBL (histologically or cytologically confirmed in $61.4 \%$ of cases) at six local district or regional hospitals in Uganda, Tanzania, and Kenya [19]. EMBLEM also enrolled healthy children from 300 random villages in the same regions as the cases [19]. To ensure the comparability of malaria exposure before enrolment for the controls and eBL cases, eligibility was restricted to usual residents $(\geq 4$ months prior to enrollment) of the study area. Malaria was assessed on venous blood specimens using light microscopy to detect asexual malaria parasite forms and commercial malaria rapid diagnostic tests (RDTs) to detect histidine rich protein-2 (HRP-2) and pan-lactate dehydrogenase (panLDH) malaria antigens $[20,21]$, which remain detectable for 35-42 days after treatment of symptomatic malaria [22]. Asexual parasites were quantified against 200 white blood cells and normalized to parasites per $\mu \mathrm{L}$ of blood.

Secondary data on children with eBL or clinical malaria were compiled from published papers by searching PubMed and Google Scholar to identify representative papers about eBL and malaria conditions. Malaria conditions in these papers were classified according to the World Health Organization algorithm into asymptomatic parasitaemia/antigenaemia or clinical malaria with six conditions (severe malaria anaemia, hyperparasitaemia, cerebral malaria, malaria prostration, moderate malaria, and mild malaria; Fig. 1) [18]. The articles were identified using the following search terms applied in different combinations: Burkitt lymphoma, severe malaria, uncomplicated malaria, symptoms, malaria progression, coma, hyperparasitaemia, prostration, haemoglobin, platelets, parasite count, sub-Saharan Africa, anaemia, malaria, and country names: Uganda, Tanzania, and Kenya. The identified articles were retrieved and screened by LSR, who read the titles, abstracts, and when this was not sufficient, read the full text to identify reports with quantitative data about eBL or malaria conditions. Case reports, case series, or viewpoints were excluded. The papers were 


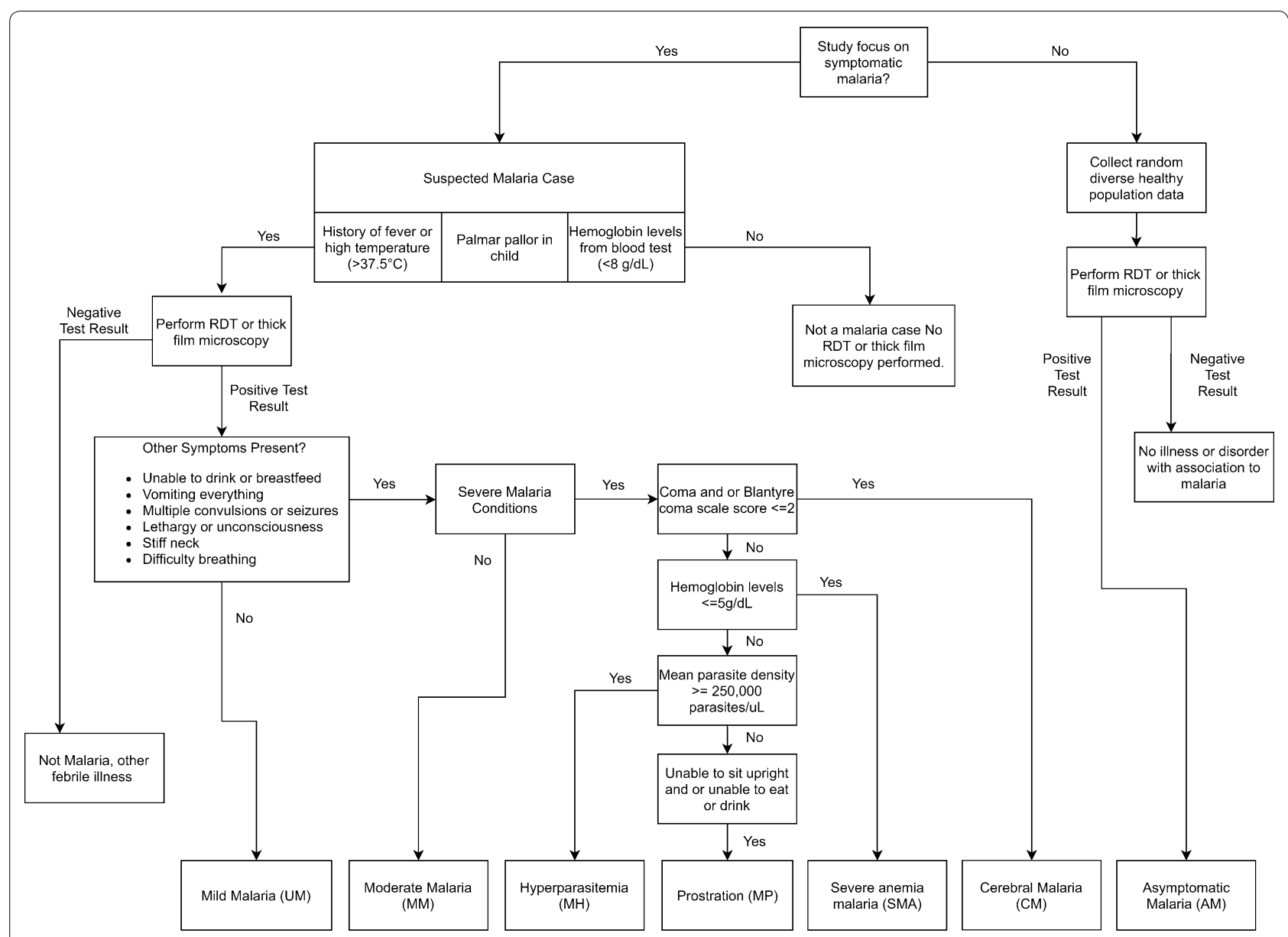

Fig. 1 Flowchart of the stepwise evaluation of different malaria conditions

prioritized according to sample size, detail and completeness of data, and whether reporting data from Uganda, Tanzania, and Kenya. This list was reviewed by LSR and SMM to select, by consensus, representative papers for data extraction.

The selected papers were used as a source of data about children with eBL, asymptomatic parasitaemia/antigenaemia, and clinical malaria. The data elements, including demographics (age, gender, and mortality), symptoms (seizures, headaches, weakness, diarrhoea, myalgia, and vomiting), signs (fever, cough, coma, hepatosplenomegaly, jaundice, pallor, and respiratory distress), and malaria-related laboratory measures (complete blood count, including haemoglobin, platelets count, WBC count; parasite density, and malaria parasitaemia based on microscopy of thick blood smears or antigenaemia based on RDT), were extracted into a spreadsheet. Malaria parasite density $>250,000$ parasites $/ \mu \mathrm{L}$ of blood was used to define hyperparasitaemia; haemoglobin $<5 \mathrm{~g} / \mathrm{dL}$ of blood was used to define severe malaria anaemia [18]; thrombocytopenia was defined as platelet counts $<150,000 / \mu \mathrm{L}$ [3]; while WBC count $>10,000$ cells/ $\mu \mathrm{L}$ was used to define leukocytosis, a general marker of inflammation.

\section{Statistical analysis}

Results expressed on a continuous scale were extracted as means and standard deviations or as medians and interquartile ranges (IQR). Medians were statistically converted into means using standard statistical methods [23] to enable all comparisons to be made with means as the measure of central tendency. The results were checked for outlier values, which were excluded as appropriate. Results expressed as categories were extracted as the percent of subjects with values falling within a certain range. Data were analysed using R 3.6.0 and R Studio 1.2.1335. The patterns of mean age and the means of malariarelated laboratory measures in children with eBL, asymptomatic parasitaemia/antigenaemia, or clinical malaria were assessed descriptively. Regression and spline models were run to explore trends of parasite density, haemoglobin, platelet count, and WBC count across single-year age 
groups in children with eBL and asymptomatic malaria parasitaemia/antigenaemia in the EMBLEM study. Twosided $P<0.05$ with Bonferroni adjustment for the number of comparisons performed were considered statistically significant.

\section{Results}

\section{Summary characteristics of study population}

Results were available for $850 \mathrm{eBL}$ cases and 2878 healthy controls with primary data in the EMBLEM study and 4019 eBL cases and 37,089 of 80,532 children with secondary data in 21 published articles selected from 685 articles had asymptomatic malaria infection or clinical malaria and their data were extracted (Fig. 2; Additional file 1: Table S1). Table 1 summarizes the patterns of demographics, parasite density, haemoglobin, platelet count, and WBC count for children with eBL, asymptomatic parasitaemia/antigenaemia, or six clinical malaria conditions. Children with eBL and asymptomatic parasitaemia/antigenaemia were similar in terms of their mean age (7.1-7.2 vs. 7.4-9.8 years), mean haemoglobin (10.110.4 vs. $11.7-12.3 \mathrm{~g} / \mathrm{dL}$ ), average malaria parasitaemia/ antigenaemia prevalence $(26.0-46.0 \%$ vs. $38.4-43.8 \%)$, parasite density ( 2800 vs. $1827-7780$ parasites $/ \mu \mathrm{L}$ ), mean platelet count $(347,000-353,000$ vs. $244,000-306,000$ platelets/ $\mu \mathrm{L})$, and WBC count $(8180-8890$ vs. $7100-7410$ cells $/ \mu \mathrm{L}$ ). Children with eBL and asymptomatic malaria parasitaemia/antigenaemia were markedly different from those with all clinical malaria conditions, who were much younger, with their mean age below age 4 years, and had markedly abnormal malaria-related laboratory results (Table 1). Children with severe malaria anaemia and malaria hyperparasitaemia had a mean age less than 2 years; those with moderate malaria, malaria prostration, and cerebral malaria had a mean age between 2 to 3 years; and those with mild malaria had a mean age of 3.2 years. Furthermore, while $75 \%$ of children with severe anaemia or moderate malaria, $65 \%$ of those with malaria prostration, and $50 \%$ of those hyperparasitaemia, cerebral malaria, or mild malaria were in children aged 0 to 2 years, only $0.1 \%$ of children with eBL and $2.4 \%$ of children with asymptomatic parasitaemia/antigenaemia were in children in this age group. However, in contrast to malaria conditions in which the male-to-female ratio was similar for children with malaria (48\% to $57 \%$ of children were male), those with eBL showed male predominance (61-63\% were male).

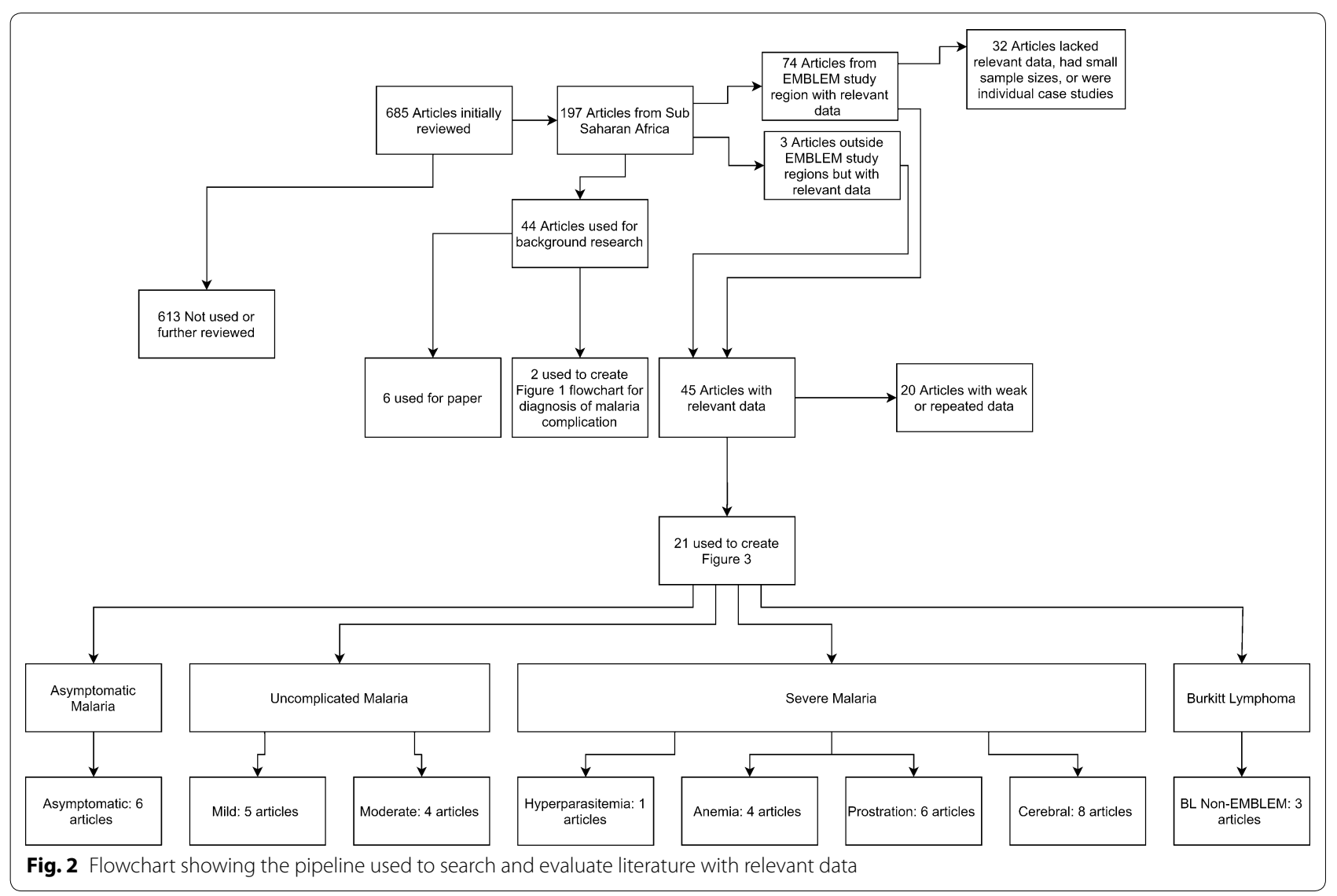




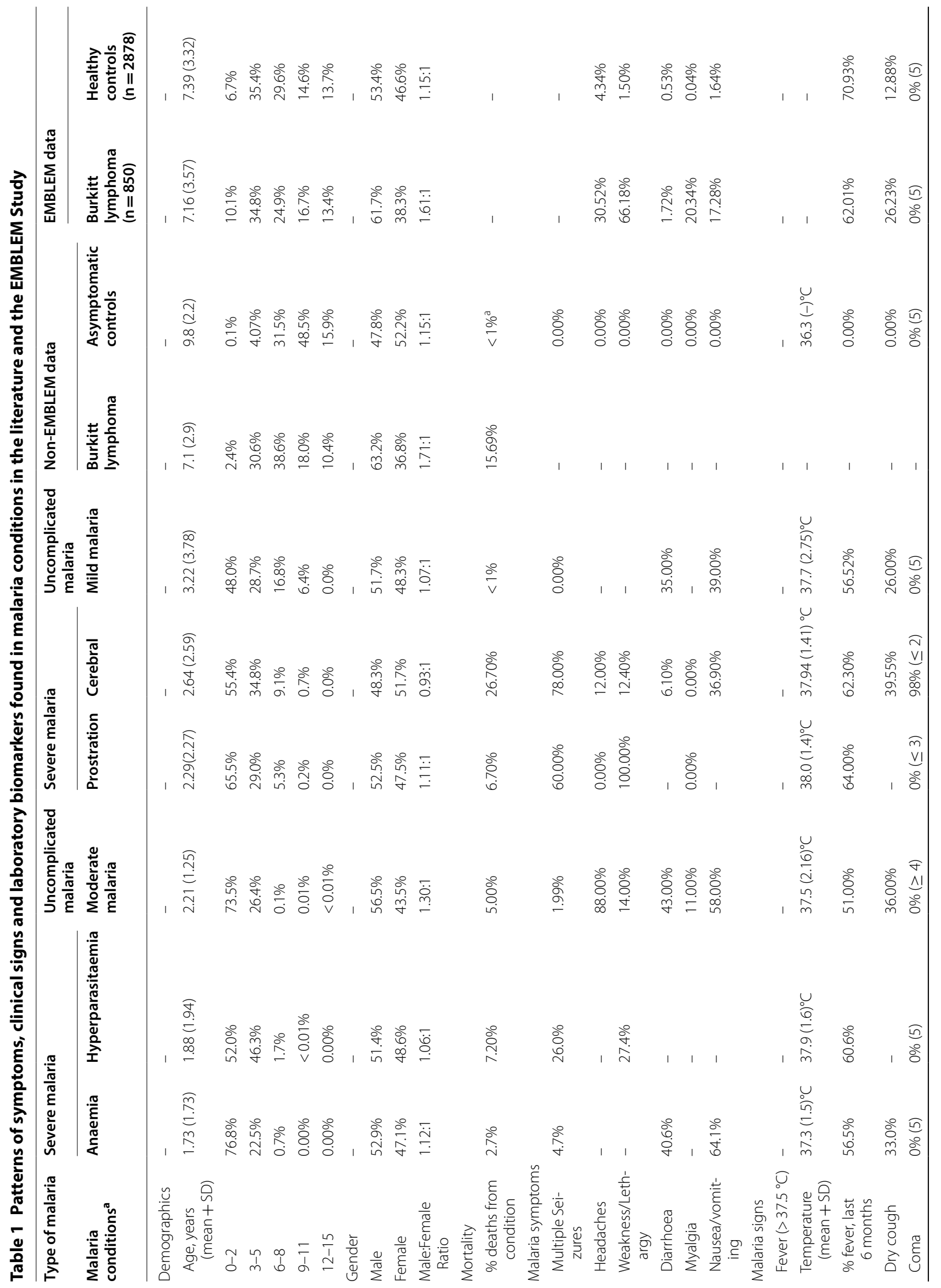




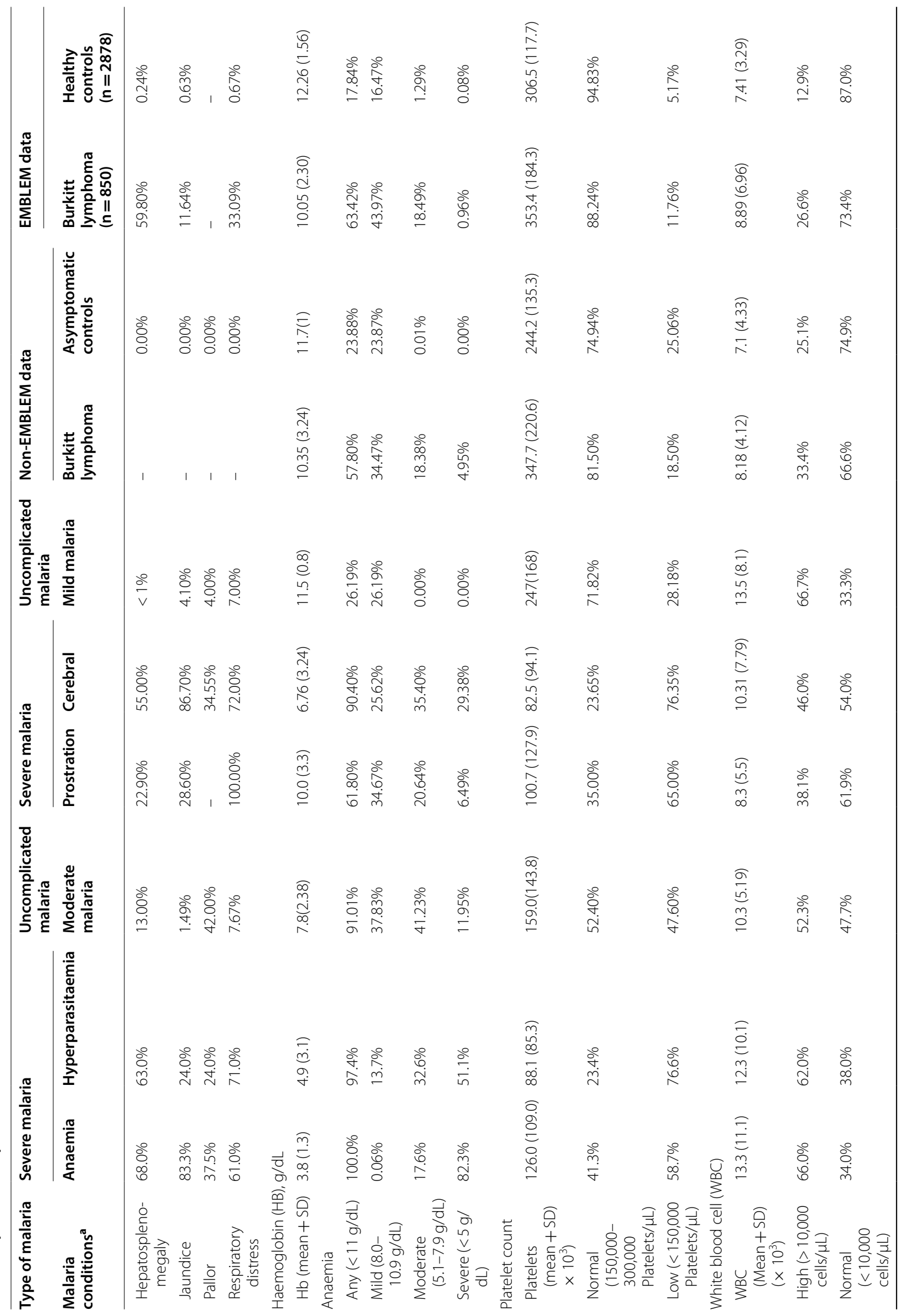




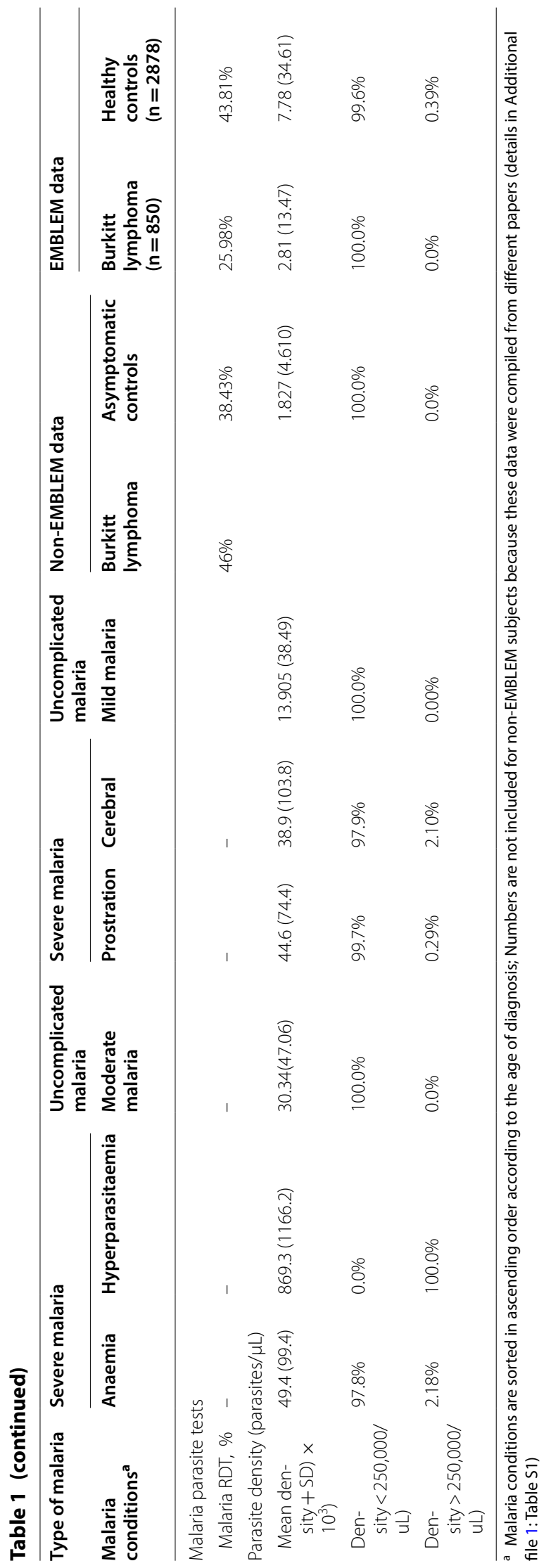




\section{Malaria-related laboratory results differ by age group and condition}

Malaria-related laboratory results showed distinct clusters in children below or above 5 years and by malaria condition (Fig. 3a-d). The children with eBL and asymptomatic parasitaemia/antigenaemia clustered above 5 years and has comparable average parasite density $<10,000$ parasites/ $\mu \mathrm{L}$ (Fig. 3a), haemoglobin $\geq 10.0 \mathrm{~g} /$ dL (Fig. 3b), platelet counts between 240,000 and 350,000 platelets $/ \mu \mathrm{L}$ (Fig. 3c) and WBC count $<10,000 \mathrm{WBCs} /$ $\mu \mathrm{L}$ (Fig. 3d). By contrast, the other malaria conditions clustered below 5 years and formed several sub-clusters. Considering parasite density, children with severe malaria anaemia, cerebral malaria, malaria prostration, moderate malaria, and mild malaria had more comparable parasite density between 10,000 to 100,000 parasites $/ \mu \mathrm{L}$, while those with hyperparasitaemia clustered apart with $>250,000$ parasites $/ \mu \mathrm{L}$ (Fig. 3a). Considering haemoglobin, three sub-clusters were apparent (Fig. 3b): children with mild malaria with haemoglobin of $>10 \mathrm{~g} /$ $\mathrm{dL}$; those with cerebral and moderate malaria with haemoglobin of $>5 \mathrm{~g} / \mathrm{dL}$ but $<10 \mathrm{~g} / \mathrm{dL}$; and those with severe anaemia and hyperparasitaemia with haemoglobin $<5 \mathrm{~g} /$ $\mathrm{dL}$. When platelet counts were considered, three subclusters were observed (Fig. 3c). The children with severe anaemia, hyperparasitaemia, prostration, or cerebral malaria clustered below 150,000 platelets $/ \mu \mathrm{L}$; those with moderate malaria clustered around $\sim 150,000 / \mu \mathrm{L}$ and those with mild malaria clustered $\sim 250,000$ platelets/ $\mu \mathrm{L}$. Considering leukocytosis ( $>10,000$ white blood cell count $/ \mu \mathrm{L}$ ), two sub-clusters of severe anaemia, hyperparasitaemia, mild malaria with WBC counts $>12,000$ cells/ $\mu \mathrm{L}$; those with cerebral malaria and moderate malaria with WBC counts with $\sim 10,000$ cells $/ \mu \mathrm{L}$, and those with prostration with WBC counts $<10,000$ cells $/ \mu \mathrm{L}$.

\section{Trends in malaria-related laboratory results across single year ages groups}

Trends in parasite density, haemoglobin, platelet count, and WBC count across single year age groups in children with eBL or asymptomatic parasitaemia/antigenaemia in the EMBLEM study are shown in Fig. 4. Parasite density increased rapidly to peak around age 4 to 5 years in children with eBL and asymptomatic parasitaemia/ antigenaemia, then decreased steadily thereafter in both groups (Fig. 4a). However, parasite density was significantly lower in children with eBL than those with asymptomatic parasitaemia/antigenaemia across all ages, with the difference being greatest in children below 5 years. The average haemoglobin was lower in children with eBL than those with asymptomatic parasitaemia/antigenaemia, but did not fall below $9 \mathrm{~g} / \mathrm{dL}$ in the eBL cases and $11 \mathrm{~g} / \mathrm{dL}$ in the children with asymptomatic parasitaemia/ antigenaemia even in the youngest age group and it increased steadily with age (Fig. 4b). When platelet counts were considered, the count was greater than 300,000 platelets $/ \mu \mathrm{L}$ in children with $\mathrm{eBL}$ and those with asymptomatic parasitaemia/antigenaemia, but the values being higher in the former than the later group (Fig. 4c). The WBC counts were generally similar in both groups, except in children below age 5 years in whom counts were slightly higher in the children with eBL (Fig. 4d).

\section{Overlap patterns of children with eBL, asymptomatic, and clinical malaria conditions}

Figure 5 summarizes the two overlap patterns showing that children with eBL and asymptomatic parasitaemia/ antigenaemia cluster together in children $>5$ years and that both groups are characterized by normal or near normal laboratory values (low parasite density, mild anaemia, and normal platelet and WBC counts). Conversely, children with clinical malaria conditions cluster together below 5 years in three sub-clusters characterized by: (a) those with severe malaria anaemia and hyperparasitaemia with markedly abnormal values of parasite density, haemoglobin, platelet count, and WBC count; (b) those with cerebral malaria and moderate malaria characterized by abnormal values of platelet count and either parasite density or haemoglobin; (c) those with malaria prostration and mild malaria characterized by abnormal values of platelet count or WBC count.

\section{Discussion}

The current study was done to evaluate whether eBL risk might be connected with high parasite density using acute malaria conditions as surrogates of moderate or high parasite density. The results show clustering of eBL and asymptomatic parasitaemia/antigenaemia in children aged $>5$ years who have normal or near normal values of parasite density, haemoglobin, platelet, and WBC counts. These results suggest that eBL could be a complication of asymptomatic parasitaemia/antigenaemia and cast doubt on the idea that it is a complication of high parasite density or the associated high inflammation, as was originally hypothesized. High parasite density was a feature of three sub-clusters of clinical malaria, all found in children below 5 years. These early age-onset malaria syndromes were characterized by markedly abnormal values of parasite density, haemoglobin, platelet counts and WBC counts. These syndromes included children with severe malaria anaemia and hyperparasitaemia, those with cerebral malaria and moderate malaria, and those with malaria prostration and mild malaria and markedly abnormal values of some or all of parasite density, haemoglobin, platelet counts and WBC counts. The findings in this study are likely valid because $\sim 9.5 \%$ of 


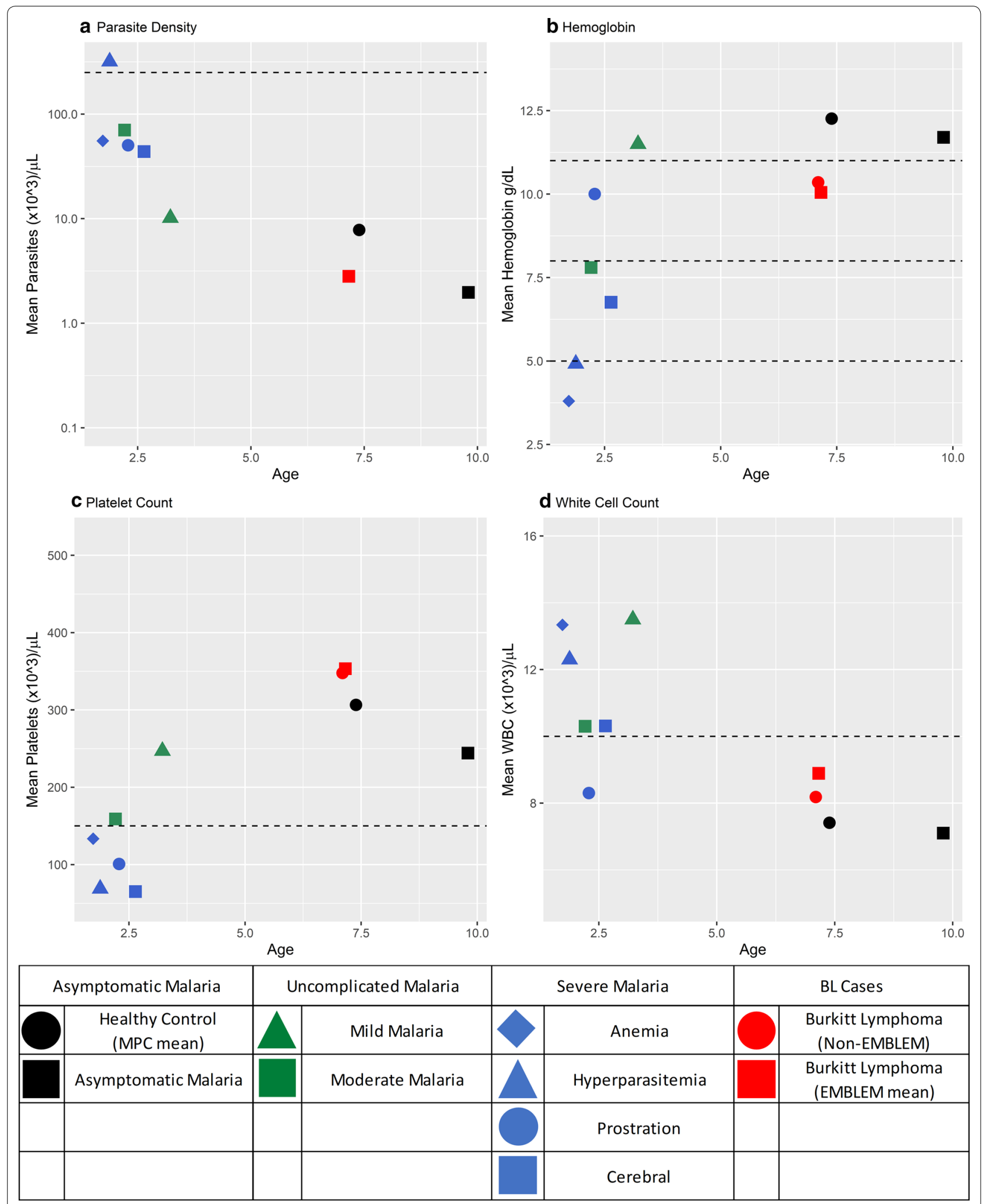

Fig. 3 Multi-panel graph showing the mean values of malaria-related laboratory measures (parasite density, haemoglobin, platelet count, and white cell count) plotted according to the mean age of the malaria condition using data obtained from the literature and the EMBLEM Study. Dotted lines mark cut-off value for hyper-parasitaemia (a), for mild $(11.0 \mathrm{~g} / \mathrm{dL})$, moderate $(7.5 \mathrm{~g} / \mathrm{dL})$, and severe anaemia $(<5.0 \mathrm{~g} / \mathrm{dL})(\mathbf{b})$, for the lower range of platelet counts (c), and for elevated white blood cell count (d) 


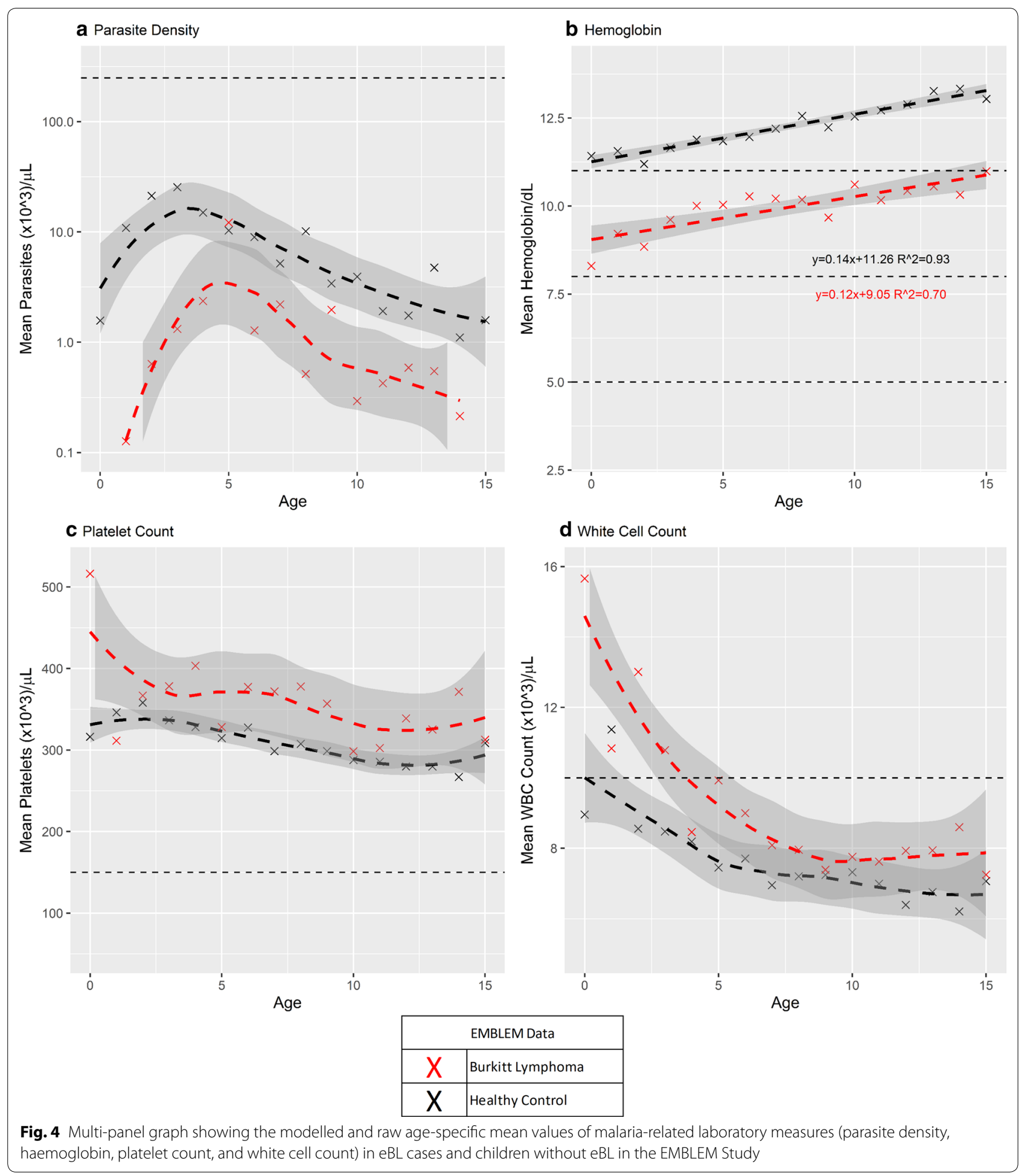

eBL cases and $6.7 \%$ of the controls in the EMBLEM study were $0-3$ years, but even in this age range the children with eBL and asymptomatic parasitaemia/antigenaemia still showed malaria-related laboratory measures that were different from those in children with acute malaria conditions. Moreover, children with eBL had significantly lower parasite density than those with asymptomatic parasitaemia/antigenaemia suggesting that the similarity 


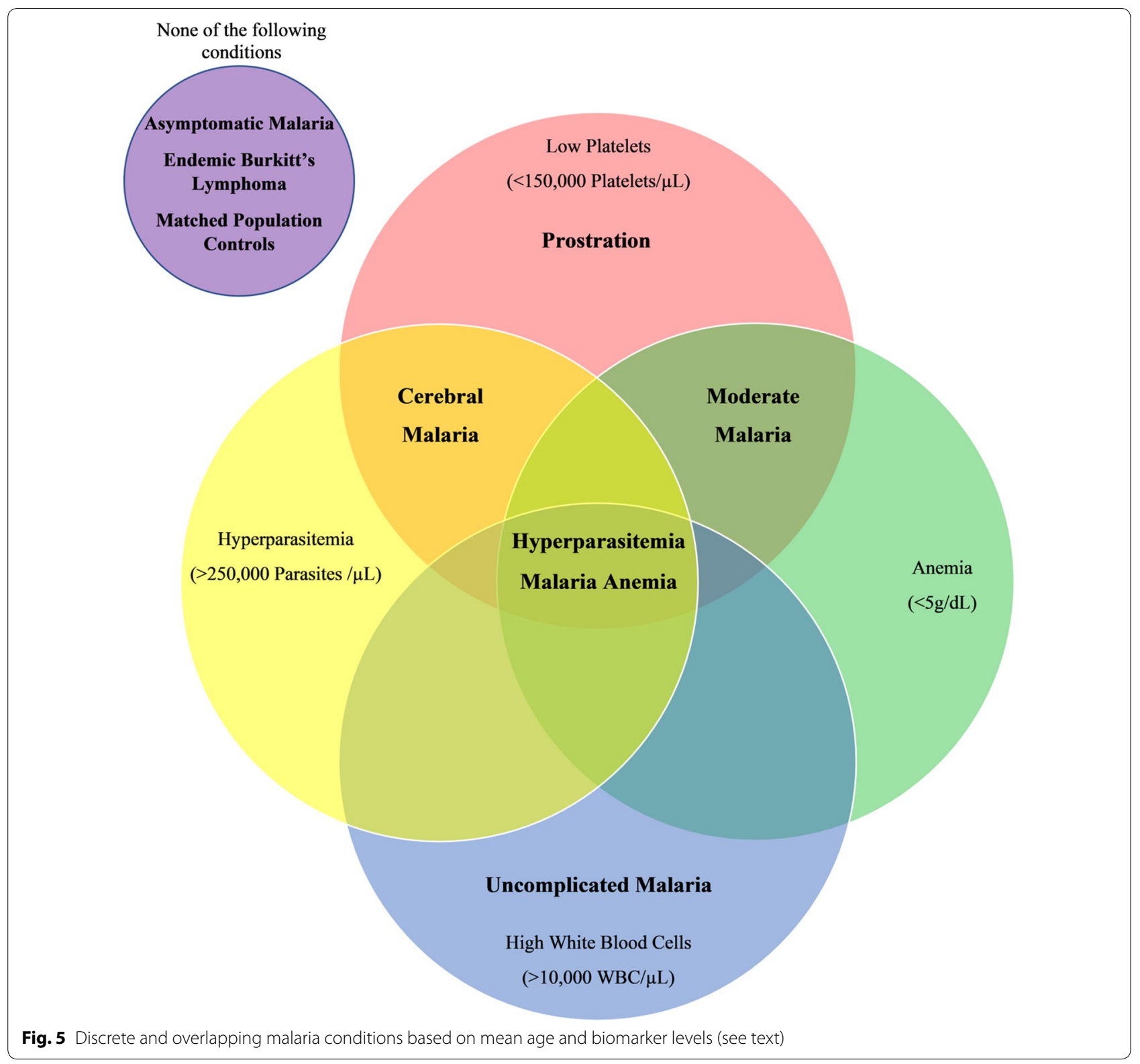

of eBL with asymptomatic parasitaemia/antigenaemia observed in overall results is established at a very early age. These results are consistent with a recent review by Quintana et al. [24] where they propose an explanation of how malaria exposure may precipitate the malignant transformation of a B-cell clone that may progress to eBL. Together, the accumulating evidence suggests that eBL develops in children who control malaria parasitaemia well, perhaps due to acquired age-related immunity to malaria [25], and that the capacity to control malaria parasitaemia precedes and continues after eBL onset.

Antigenic stimulation from malaria is widely accepted as an insult that leads to $B$ cell proliferation in the germinal center and increases the chance of translocation of $c-M Y C$ into the vicinity of immunoglobulin enhancer elements and progression to eBL [26]. However, it has remained unclear whether uncontrolled malaria parasite proliferation, i.e., high parasite density, plays a key part in antigenic stimulation from malaria that triggers eBL development. The analysis presented suggests otherwise because asymptomatic parasitaemia/antigenaemia is associated with well-controlled malaria infection. If so, then children with eBL have a well-controlled malaria infection at the time of their diagnosis. The results from EMBLEM are notable because previous analyses suggest that $\mathrm{eBL}$ cases were more likely to be exposed to 
heavy malaria, based on being more likely to live in a village near surface water, to report inpatient or outpatient malaria history $>13$ months before enrollment, than geographically matched controls [12], thereby underscoring the likelihood that immunity to malaria is likely to develop prior to eBL onset. The current analysis suggests that immunity to malaria appears to be established from a young age because children below 5 years with eBL had lower haemoglobin than children with asymptomatic malaria, consistent with heavy exposure to malaria, but significantly lower parasite density, consistent with a capacity to control malaria parasitaemia in young children. In view of the fact that malaria is the pre-eminent cause of early mortality in areas where eBL is common [17], the overlap patterns observed support a speculation that eBL occurs in children who are adapted to heavy exposure to malaria and it may be a trade-off exchange for a high risk for death from acute malaria. Because children with eBL are less likely to carry the classical polymorphisms that protect from severe malaria [10-12], such as the sickle cell trait [12], the trade-off for the much rarer, albeit deadly at the individual level, like involves mechanisms of resistance to early malaria mortality that are currently unknown but worth investigating [12].

Asymptomatic malaria is typically associated with low-, rather than high-parasite density malaria parasitaemia. Thus, these results also suggest that eBL risk may be associated with low density malaria infection. Furthermore, because an asymptomatic infection, particularly in children above 5 years, is also likely to be a clonally diverse infection [27], these results raise the hypothesis that a clonally diverse malaria antigenic stimulation is related to eBL development. This hypothesis is consistent with previous findings that concurrent infection with multiple malaria genotypes was correlated with eBL [28] and that eBL cases were more likely to have a higher $P$. falciparum genetic diversity score than controls [29]. This hypothesis is also consistent with findings in the EMBLEM study that children with eBL were less likely to report clinical malaria up to 12 months before eBL diagnosis and less likely to have detectable malaria parasite/antigens at enrolment [19] because children aged $>5$ years with clonally diverse malaria infection had a decreased risk for clinical malaria during a follow up of 90 or more days [30]. The hypotheses that parasite density and genetic diversity are related to eBL could be tested in the era of next generation sequencing or proteomic technologies $[28,29]$.

This study has some limitations, notably, reliance on cross-sectional data. Thus, the correlations should not be interpreted as evidence for causality. Second, the reliance on published literature to increase complement assessment in EMBLEM is a strength, but the literature may be biased or incomplete. For example, studies of asymptomatic parasitaemia/antigenaemia may under-sample children below 5 years, or studies of severe malaria may oversample children below 5 years, which would lead to erroneous mean age patterns. However, the results from the EMBLEM study, which enrolled representative, population-based eBL cases and controls and uniformly tested subjects for malaria-related laboratory measures, support the overall patterns. This study lacks direct measures of immunity to malaria, including anti-malaria antibodies, which limits the ability to compare the immune status of eBL cases with that of children with asymptomatic parasitaemia/antigenaemia or clinical malaria. The strengths of this study include the large sample size and detailed data from the EMBLEM study and published data about children with malaria from malaria endemic areas.

\section{Conclusions}

The findings suggest that children with eBL and asymptomatic parasitaemia/antigenaemia were most similar with respect to age and malaria-related laboratory results and that both groups were markedly different from children with clinical malaria. These results raise a testable hypothesis that asymptomatic parasitaemia/antigenaemia, and by extension its associated low malaria parasite density, may be the antigenic stimulation from malaria related to eBL onset.

\section{Supplementary information}

Supplementary information accompanies this paper at https://doi. org/10.1186/s12936-020-03312-7.

Additional file 1: Table S1. A list of articles with used as sources for relevant data about malaria conditions in East Africa.

\section{Abbreviations}

OR: Odds ratio; Cl: Confidence interval; eBL: Endemic Burkitt lymphoma; WBC: White blood cell; EMBLEM study: Epidemiology of Burkitt Lymphoma in East African Children and Minors Study; HRP-2: Histidine rich protein-2; pan-LDH: Pan-lactate dehydrogenase.

\section{Acknowledgements}

We thank the study population and communities for their participation. We are grateful to Mr. Josiah Magatti of Shirati Health Education and Development Foundation for his contribution to the design of the EMBLEM study, securing ethical permission to conduct the study in Tanzania, and leading the implementation of fieldwork in Tanzania up to the time of his passing in 2014. We thank Ms. Janet Lawler-Heavner at Westat Inc, (Rockville, MD, USA) and Mr. Erisa Sunday at the African Field Epidemiology Network (Kampala, Uganda) for managing the study. We are grateful to the leadership of the collaborating countries and institutions for hosting local field offices and laboratories and supporting the fieldwork. We thank Ms. Laurie Buck, Dr. Carol Giffen, Mr. Greg Rydzak and Mr. Jeremy Lyman at Information Management Services Inc. (Calverton, MD, USA) for coordinating data, and preparing data analysis files.

\section{Authors' contributions}

LSR, SMM designed the study and led the analysis of this study. SMM, MDO, PK, SJR, CNT, PAW, RTK, NM, EK, KB, LWA, and JJG provided administrative and fieldwork oversight for the EMBLEM study. IO, IDL, and DH conducted and 
monitored field work. LSR drafted the manuscript. All authors contributed to the manuscript. All authors read and approved the final manuscript.

\section{Funding}

This study was funded by the Intramural Research Program of the Division of Cancer Epidemiology and Genetics, National Cancer Institute (NCl) (Contracts HHSN261201100063C and HHSN261201100007I) and, in part, by the Intramural Research Program, National Institute of Allergy and Infectious Diseases (SJR), National Institutes of Health, Department of Health and Human Services. The content of this publication does not necessarily reflect the views or policies of the Department of Health and Human Services, nor does mention of trade names, commercial products, or organizations imply endorsement by the US Government.

\section{Availability of data}

The data and the code used for these analyses are available on reasonable requests from the corresponding authors.

\section{Ethics approval and consent to participate}

Ethical approval to conduct the EMBLEM study was given by the Uganda Virus Research Institute Research and Ethics Committee, the Uganda National Council of Science and Technology (HS-816), and the National Cancer Institute Special Studies Institutional Review Board (10-C-N133).

\section{Consent to participate}

Written informed consent was obtained from the parents or guardians of children in EMBLEM and written informed assent was obtained from children in EMBLEM aged 7 years or older prior to enrolment.

\section{Consent for publication}

The manuscript does not include details, images, or videos relating to an individual person. All data are presented in aggregate and are not identifiable at the individual level.

\section{Competing interests}

The authors declare that they have no competing interests.

\section{Author details}

${ }^{1}$ Division of Cancer Epidemiology and Genetics, National Cancer Institute, National Institutes of Health, Bethesda, MD, USA. ${ }^{2}$ EMBLEM Study, St. Mary's Hospital Lacor, Gulu, Uganda. ${ }^{3}$ African Field Epidemiology Network, Kampala, Uganda. ${ }^{4}$ EMBLEM Study, Kuluva Hospital Kuluva, Arua, Uganda. ${ }^{5}$ Division of Intramural Research, National Institute of Allergy and Infectious Diseases, National Institutes of Health, Bethesda, MD, USA. ${ }^{6}$ EMBLEM Study, Moi University College of Health Sciences, Eldoret, Kenya. ${ }^{7}$ EMBLEM Study, Academic Model Providing Access to Healthcare (AMPATH), Eldoret, Kenya. ${ }^{8}$ EMBLEM Study, Bugando Medical Center, Mwanza, Tanzania. ${ }^{9}$ EMBLEM Study, Shirati Health and Educational Foundation, Shirati, Tanzania. ${ }^{10}$ Department of Pathology, The Ohio State University, Columbus, OH, USA.

Received: 21 March 2020 Accepted: 30 June 2020 Published online: 28 July 2020

\section{References}

1. Burkitt DP. Etiology of Burkitt's lymphoma-an alternative hypothesis to a vectored virus. J Natl Cancer Inst. 1969;42:19-28.

2. Rainey JJ, Omenah D, Sumba PO, Moormann AM, Rochford R, Wilson ML. Spatial clustering of endemic Burkitt's lymphoma in high-risk regions of Kenya. Int J Cancer. 2007;120:121-7.

3. Kafuko GW, Burkitt DP. Burkitt's lymphoma and malaria. Int J Cancer. 1970;6:1-9.

4. Parkin DM, Sitas F, Chirenje M, Stein L, Abratt R, Wabinga H. Part I: cancer in indigenous Africans-burden, distribution, and trends. Lancet Oncol. 2008;9:683-92.

5. Hammerl L, Colombet M, Rochford R, Ogwang DM, Parkin DM. The burden of Burkitt lymphoma in Africa. Infect Agent Cancer. 2019;14:17.

6. Aka P, Vila MC, Jariwala A, Nkrumah F, Emmanuel B, Yagi M, et al. Endemic Burkitt lymphoma is associated with strength and diversity of Plasmodium falciparum malaria stage-specific antigen antibody response. Blood. 2013;122:629-35.

7. Mutalima N, Molyneux E, Jaffe H, Kamiza S, Borgstein E, Mkandawire $\mathrm{N}$, et al. Associations between Burkitt lymphoma among children in Malawi and infection with HIV, EBV and malaria: results from a casecontrol study. PLOS ONE. 2008;3:e2505.

8. Carpenter LM, Newton R, Casabonne D, Ziegler J, Mbulaiteye S, Mbidde E, et al. Antibodies against malaria and Epstein-Barr virus in childhood Burkitt lymphoma: a case-control study in Uganda. Int J Cancer. 2008;122:1319-23.

9. Guech-Ongey M, Yagi M, Palacpac NM, Emmanuel B, Talisuna AO, Bhatia K, et al. Antibodies reactive to Plasmodium falciparum serine repeat antigen in children with Burkitt lymphoma from Ghana. Int J Cancer. 2012;130:1908-14.

10. Williams $A O$. Haemoglobin genotypes, $A B O$ blood groups, and Burkitt's tumour. J Med Genet. 1966;3:177-9.

11. Hesseling PB, Jam DT, Palmer DD, Wharin P, Tuh GS, Bardin R, et al. Burkitt's lymphoma patients in Northwest Cameroon have a lower incidence of sickle cell trait (Hb AS) than healthy controls. S Afr Med J. 2016;106:10693.

12. Legason ID, Pfeiffer RM, Udquim KI, Bergen AW, Gouveia MH, Kirimunda $S$, et al. Evaluating the causal link between malaria infection and endemic Burkitt Lymphoma in Northern Uganda: a Mendelian randomization study. EBioMedicine. 2017;25:58-65.

13. Jallow M, Teo YY, Small KS, Rockett KA, Deloukas P, Clark TG, et al. Genome-wide and fine-resolution association analysis of malaria in West Africa. Nat Genet. 2009;41:657-65.

14. Pike MC. Burkitt's lymphoma and sickle-cell trait in Uganda. Br J Prev Soc Med. 1970;24:63.

15. Nkrumah FK, Perkins IV. Sickle cell trait, hemoglobin C trait, and Burkitt's lymphoma. Am J Trop Med Hyg. 1976;25:633-6.

16. Mulama DH, Bailey JA, Foley J, Chelimo K, Ouma C, Jura WG, et al. Sickle cell trait is not associated with endemic Burkitt lymphoma: an ethnicity and malaria endemicity-matched case-control study suggests factors controlling EBV may serve as a predictive biomarker for this pediatric cancer. Int J Cancer. 2014;134:645-53.

17. Marsh K, Snow RW. Host-parasite interaction and morbidity in malaria endemic areas. Philos Trans R Soc Lond B Biol Sci. 1997;352:1385-94.

18. WHO. Guidelines for the treatment of malaria. 2nd ed. Geneva: World Health Organization; 2010.

19. Peprah S, Ogwang MD, Kerchan P, Reynolds SJ, Tenge CN, Were PA, et al. Risk factors for Burkitt lymphoma in East African children and minors: a case-control study in malaria-endemic regions in Uganda, Tanzania and Kenya. Int J Cancer. 2020;146:953-69.

20. Maziarz M, Kinyera T, Otim I, Kagwa P, Nabalende H, Legason ID, et al. Age and geographic patterns of Plasmodium falciparum malaria infection in a representative sample of children living in Burkitt lymphomaendemic areas of northern Uganda. Malar J. 2017;16:124.

21. Peprah S, Tenge C, Genga IO, Mumia M, Were PA, Kuremu RT, et al. A cross-sectional population study of geographic, age-specific, and household risk factors for asymptomatic Plasmodium falciparum malaria infection in Western Kenya. Am J Trop Med Hyg. 2019:100:54-65.

22. Grandesso F, Nabasumba C, Nyehangane D, Page AL, Bastard M, De Smet $M$, et al. Performance and time to become negative after treatment of three malaria rapid diagnostic tests in low and high malaria transmission settings. Malar J. 2016;15:496.

23. Wan X, Wang W, Liu J, Tong T. Estimating the sample mean and standard deviation from the sample size, median, range and/or interquartile range. BMC Med Res Methodol. 2014;14:135.

24. Quintana MDP, Smith-Togobo C, Moormann A, Hviid L. Endemic Burkitt lymphoma-an aggressive childhood cancer linked to Plasmodium falciparum exposure, but not to exposure to other malaria parasites. APMIS. 2020;128:129-35.

25. Rodriguez-Barraquer I, Arinaitwe E, Jagannathan P, Boyle MJ, Tappero J, Muhindo M, et al. Quantifying heterogeneous malaria exposure and clinical protection in a cohort of Ugandan children. J Infect Dis. 2016;214:1072-80.

26. Rochford R, Cannon MJ, Moormann AM. Endemic Burkitt's lymphoma: a polymicrobial disease? Nat Rev Microbiol. 2005;3:182-7. 
27. Doolan DL, Dobano C, Baird JK. Acquired immunity to malaria. Clin Microbiol Rev. 2009;22:13-36.

28. Emmanuel B, Kawira E, Ogwang MD, Wabinga H, Magatti J, Nkrumah F, et al. African Burkitt lymphoma: age-specific risk and correlations with malaria biomarkers. Am J Trop Med Hyg. 2011;84:397-401.

29. Johnston WT, Mutalima N, Sun D, Emmanuel B, Bhatia K, Aka P, et al. Relationship between Plasmodium falciparum malaria prevalence, genetic diversity and endemic Burkitt lymphoma in Malawi. Sci Rep. 2014;4:3741.
30. Eldh M, Hammar U, Arnot D, Beck HP, Garcia A, Liljander A, et al. Multi plicity of asymptomatic Plasmodium falciparum infections and risk of clinical malaria: a systematic review and pooled analysis of individual participant data. J Infect Dis. 2020;221:775-85.

\section{Publisher's Note}

Springer Nature remains neutral with regard to jurisdictional claims in published maps and institutional affiliations.
Ready to submit your research? Choose BMC and benefit from:

- fast, convenient online submission

- thorough peer review by experienced researchers in your field

- rapid publication on acceptance

- support for research data, including large and complex data types

- gold Open Access which fosters wider collaboration and increased citations

- maximum visibility for your research: over $100 \mathrm{M}$ website views per year

At BMC, research is always in progress.

Learn more biomedcentral.com/submissions 